Open Peer Review on Qeios

\title{
The link between clinical, biological and metabolic factors in patients with metastatic renal cell carcinoma (mRCC) treated with immunotherapy
}

Antonio Russo

Funding: The author(s) received no specific funding for this work.

Potential competing interests: The author(s) declared that no potential competing interests exist.

\section{Abstract}

Lorena Incorvaia*2 ${ }^{\text {Chiara Brando}} \underline{1}$, Fanale Daniele ${ }^{1}$, Algeri Laura ${ }^{1}$, Scalia Raimondo ${ }^{1}$, Dimino Alessandra ${ }^{1}$, Bonasera Annalisa ${ }^{1}$, Fiorino Alessia ${ }^{1}$, Pedone Erika ${ }^{1}$, Bono Marco ${ }^{1}$, Barraco Nadia ${ }^{1}$, Galvano Antonio $^{1}$, Gristina Valerio ${ }^{1}$, Corsini Lidia Rita ${ }^{1}$, Badalamenti Giuseppe ${ }^{1}$, Bazan Viviana ${ }^{2}$, Russo Antonio ${ }^{1}$

${ }^{1}$ Department of Surgical, Oncological and Oral Sciences, Section of Medical Oncology, University of Palermo, 90127 Palermo, Italy.

${ }^{2}$ Department of Biomedicine, Neuroscience and Advanced Diagnostics (Bi.N.D.), Section of Medical Oncology, University of Palermo, 90127 Palermo, Italy.

\section{Introduction}

Renal cell carcinoma $(R C C)$ is a heterogeneous group of cancer subtypes with different morphological and genetic features, molecular pathogenesis, and clinical behavior (1). It accounts for approximately $75 \%$ of all renal cancers' and represents a tumor where the new biological and immunological knowledge have changed the clinical landscape and natural history of the disease (1).

The introduction of checkpoint inhibitors has resulted in durable responses and improvements in overall survival (OS) in patients with advanced RCC (2-5), but treatment efficacy is widely variable ranging from rapid disease progression to sustained complete remissions and a substantial number of patients are resistant to PD- Inhibition 1/PD-L1 showing an overall low response rate $(3,6)$. Biological factors specific to some individuals have a clear effect on this variation in response. Predicting which patient will benefit from immunotherapy still remains a problem, as well as an unmet need (1) (Figure 1).

This variability in clinical response to immunotherapy requires the discovery of predictive biomarkers that 
could help identify patient subgroups for immune checkpoint treatment.

Although biomarkers continue to evolve, to identify patients who will likely benefit from therapy is poorly predictable in daily clinical practice. Consortium (IMDC) risk score or Heng criteria remain the main criteria used in clinical practice, and also form the basis of risk stratification for clinical trials. However, IMDC risk groups were validated in a cohort of patients treted with targeted therapy, and their prognostic and predictive power may be lower in the new treatment landscape.

Based on these evidence, we integrated IMDC risk group with different clinicle and biological variable (metastatic sites, number of metastasis, Body Mass Index (BMI), and basal plasma levels of soluble PD-1 (sPD-1), PD-L1 (sPD-L1), and BTN3A1 (sBTN3A1)) in metastatic RCC (mRCC) patients.

\section{Methods}

A retrospective study was carried out from March 2017 to January 2019 at the 'Sicilian Regional Center for the Prevention, Diagnosis and Treatment of Rare and Heredo-Familial Tumors' of the Section of Medical Oncology of University Hospital Policlinico ‘P. Giaccone’ of Palermo.

We analyzed all clinical information and collected a peripheral blood sample regarding a cohort of 41 patients with histologically confirmed diagnosis.

We integrated the IMDC with the following variables in a population of mRCC patients treated with nivolumab: metastatic sites, number of metastasis, BMI, and basal plasma levels of SPD-1, sPD-L1, and sBTN3A1 measured using homemade ELISA assays not yet commercially available, designed according to investigator specifications. To identify independent prognostic factors for Time to Treatment Failure (TTF), univariate and multivariate Cox proportional hazard regression models were built.

\section{Results}

The primary outcome investigated was TTF. The median TTF to nivolumab treatment was 22 months. Using thresholds by ROC analysis for soluble immune-checkpoints, and univariate/multivariate Cox proportional hazard regression models to statistical analysis, we found that the RCC patients with high baseline levels of sPD-1 (>2 ng/ml), and SBTN3A1 (>6.8 ng/ml), BMI>25, prognostic IMDC factor > 1, no bone and/or brain metastases, metastatic sites $\leq 2$, were associated to longer time to treatment failure. Data on overall survival (OS) are under-evaluation.

\section{Conclusions}

Although physicians are inundated from increased information related to the patients and tumor characteristics, to identify prognostic and predictive biomarkers remains an issue. The use of a composed model including clinical, but also biological and metabolic factors specific for certain individuals, could provide important contributions to the development of most accurate prognostic and predictive score. 


\section{References}

1. Incorvaia L, Fanale D, Badalamenti G, Porta C, Olive D, De Luca I, et al. Baseline plasma levels of soluble PD-1, PD-L1, and BTN3A1 predict response to nivolumab treatment in patients with metastatic renal cell carcinoma: a step toward a biomarker for therapeutic decisions. Oncoimmunology. 2020;9(1):1832348.

2. Motzer RJ, Escudier B, McDermott DF, George S, Hammers HJ, Srinivas S, et al. Nivolumab versus Everolimus in Advanced Renal-Cell Carcinoma. N Engl J Med. 2015;373(19):1803-13.

3. Motzer RJ, Tannir NM, McDermott DF, Arén Frontera O, Melichar B, Choueiri TK, et al. Nivolumab plus Ipilimumab versus Sunitinib in Advanced Renal-Cell Carcinoma. N Engl J Med. 2018;378(14):1277-90. 4. Choueiri TK, Motzer RJ, Rini BI, Haanen J, Campbell MT, Venugopal B, et al. Updated efficacy results from the JAVELIN Renal 101 trial: first-line avelumab plus axitinib versus sunitinib in patients with advanced renal cell carcinoma. Ann Oncol. 2020;31(8):1030-9.

5. Zhu X, Lang J. Soluble PD-1 and PD-L1: predictive and prognostic significance in cancer. Oncotarget. 2017;8(57):97671-82.

6. Incorvaia L, Fanale D, Badalamenti G, Brando C, Bono M, De Luca I, et al. A "Lymphocyte MicroRNA Signature" as Predictive Biomarker of Immunotherapy Response and Plasma PD-1/PD-L1 Expression Levels in Patients with Metastatic Renal Cell Carcinoma: Pointing towards Epigenetic Reprogramming. Cancers (Basel). 2020;12(11). 